\title{
Anisotropic Cosmological Models with Two Fluids
}

\author{
B. Mishra ${ }^{(D)}{ }^{1}{ }^{\text {Pratik P. Ray, }}{ }^{1}$ and S. K. J. Pacif ${ }^{2}$ \\ ${ }^{1}$ Department of Mathematics, Birla Institute of Technology and Science, Pilani, Hyderabad Campus, Hyderabad 500 078, India \\ ${ }^{2}$ Centre of Theoretical Physics, Jamia Millia Islamia, New Delhi 110 025, India \\ Correspondence should be addressed to B. Mishra; bivudutta@yahoo.com
}

Received 25 July 2017; Revised 21 October 2017; Accepted 26 December 2017; Published 23 January 2018

Academic Editor: Edward Sarkisyan-Grinbaum

Copyright (C) 2018 B. Mishra et al. This is an open access article distributed under the Creative Commons Attribution License, which permits unrestricted use, distribution, and reproduction in any medium, provided the original work is properly cited. The publication of this article was funded by SCOAP ${ }^{3}$.

\begin{abstract}
Anisotropic dark energy cosmological models have been constructed in a Bianchi V space-time, with the energy momentum tensor consisting of two noninteracting fluids, namely, bulk viscous fluid and dark energy fluid. Two different models are constructed based on the power law cosmology and de Sitter universe. The constructed model was also embedded with different pressure gradients along different spatial directions. The variable equation of state (EoS) parameter and skewness parameters for both models are obtained and analysed. The physical properties of the models obtained with the use of scale factors of power law and de Sitter law are also presented.
\end{abstract}

\section{Introduction}

The most popular problem in modern cosmology has been invoked by the current discovery of accelerated expansion of the universe. This has been confirmed as an established fact through different observational data, such as Type Ia Supernovae (SNIa) [1, 2], CMB radiation [3-5], and gravitational lensing $[6,7]$. This development is explained at the backdrop of general relativity (GR) through the introduction of an unknown energy source termed as dark energy (DE). This DE provides a repulsive gravity that helps in driving the acceleration by generating a strong negative force leading to an antigravity effect. In Friedman-Robertson-Walker (FRW) universes, viscosity appears as the only dissipate phenomenon, so a considerable amount of interest is seen in the study of cosmological models with bulk viscous fluid. In the inflationary phase, the contribution of bulk viscosity is well recognized, which gives rise to a negative pressure that simulates a repulsive gravity. The equation of state (EoS) parameter of the viscous fluid, having value lower than -1 , is generally considered to be significant in the context of DE cosmology, which is also indictaed by observational results [3-5]. However, DE crossing phantom divide line having $\omega$ greater than or equal to 1 is lightly favoured. In consistency with the observational results, Copeland et al. [8] and Li et al.
[9] have used the scalar field approach with an introduction of time-dependent EoS parameter to obtain the acceptable range for $\omega$. Another way to achieve this result is to reveal the solutions of Einstein's field equations by incorporating some kinematical assumptions, which are in consistency with the observed kinematics of the universe. As a testimony to this, Hubble parameter has been widely used to obtain explicit accelerating cosmological models in the framework of spatially homogeneous space-time [10].

It can be noted that dominance of an anisotropic stress gives rise to an anisotropic expansion. This dominance will have a considerable impact via anisotropic stress on cosmological evolutions such as magnetic fields, hydrodynamic shear viscosity, and collisionless relativistic particles $[11,12]$. However, researches on DE with homogeneous and anisotropic space-time with time-varying EoS parameter observed that, at late time of cosmic evolution, $\mathrm{DE}$ yields isotropic pressure $[13,14]$. On the other hand, several researchers [15-17] focused on the fact that Wilkinson Microwave Anisotropy Probe (WMAP) data [18, 19] requires Bianchi type morphology instead of FriedmanRobertson-Walker (FRW) type for better accurate explanation of the anisotropic universe. Campanelli et al. [20] revealed that, irrespective of the level of anisotropy in geometry of the universe and dark energy EoS, the SNIa 
data are always more consistent with standard isotropic universe.

Mishra et al. [21] have constructed the cosmological model based on pressure anisotropy in the presence of a gauge function, whereas Mishra et al. [22] have studied the anisotropic universe with general forms of scale factor. Several cosmological models were obtained with constant deceleration parameter, where the matter is in the form of perfect fluid or ordinary matter. However, many of those matters are not enough to describe the dynamics of an accelerating universe relating to anisotropy. This motivates us to consider the model of the accelerating universe filled with noninteracting fluids [23-25]. Akarsu and Kilinc [26, 27] have assumed constant deceleration parameter to construct and investigate DE models in Bianchi I and Bianchi III spacetime. Yadav et al. [28] have assumed variable EoS parameter but constant deceleration parameter to construct the DE cosmological model in a locally rotationally symmetric Bianchi V space-time. Theoretical models of interacting and noninteracting DE have been discussed widely in the literature [29-31]. The paper is arranged as follows: in Section 2, a mathematical formalism of an anisotropic DE universe is presented along with the relevant physical parameters. Two dark energy cosmological models, one with power law cosmology and the other with de Sitter universe, have been constructed and analysed in Section 3. The summary is given in Section 4.

\section{Formalism}

From an observational viewpoint, one of the most important results is the theorem of Wald [32], which states that universe with accelerating expansion tends towards isotropy at late phase. As a matter of fact, if the universe undergoes an early period of inflation, the present day universe will seem to be highly isotropic. Further, since the universe has now started accelerating, any kind of anisotropy will remain small in the late phase of cosmic acceleration. Bianchi universes are the class of cosmological models that are homogeneous but not necessarily isotropic on spatial slices. It contains, as a subclass, the standard isotropic model known as FRW universe. Calculations of nucleosynthesis and microwave background anisotropies in Bianchi models have been compared against data from the real universe, typically giving null results which can be translated into upper limits on anisotropy. Tentative detections of nonzero anisotropic shear by Jaffe et al. [33] are currently believed to be in consistency with other known cosmological parameters [34] and with polarization of the microwave background [35]. However, these models remain widely studied for their pedagogical value, mainly making them exact tractable solutions of Einstein's field equation.

In the present paper, we are interested in studying the behaviour of anisotropy universe in the DE cosmological model. The standard FRW universe is homogeneous and isotropic. But, in order to address the small-scale anisotropy nature of the universe, Bianchi space-time is well accepted as it represents a globally hyperbolic spatially homogeneous but not isotropic space-time. Among all 9 space-times of Bianchi, Bianchi V space-time is very intuitive as it has more degrees of freedom characterized by Lie groups and generates pseudospherical space. Hence, in order to construct an anisotropic DE cosmological model in GR, we have considered here Bianchi $\mathrm{V}$ space-time in the form

$$
d s^{2}=d t^{2}-\sum_{i=1}^{3} e^{2 a_{i} x} b_{i}^{2} d x_{i}^{2}
$$

where $b_{i}=b_{i}(t), i=1,2,3$, are the directional scale factors considered to be different along three orthogonal directions and thereby provide a source for anisotropic expansion. Here, we choose $a_{1}=0$, and $a_{2}=a_{3}=a$, with $a$ being a nonzero arbitrary constant. Assuming that GR is well defined at cosmic scales, we incorporate Einstein's field equations,

$$
G_{i j} \cong R_{i j}-\frac{1}{2} R g_{i j}=\kappa T_{i j}
$$

where $G_{i j}, R_{i j}, R$, and $T_{i j}$, respectively, denote the Einstein tensor, Ricci tensor, Ricci scalar, and total effective energy momentum tensor (EMT) and $\kappa=8 \pi G / c^{4}$. $G$ is the Newtonian gravitational constant and $c$ is the speed of light with $8 \pi G=c=1$. Here, EMT consists of two different components: the barotropic bulk viscous fluid $\left(T_{i j}^{\mathrm{vis}}\right)$ and $\mathrm{DE}$ fluid $\left(T_{i j}^{\mathrm{de}}\right)$. In case of barotropic cosmic fluid, the proper pressure $p$ is given as $=\xi \rho(0 \leq \xi \leq 1)$. The pressure with the contribution from bulk viscosity is also directly proportional to energy density; that is, $3 \zeta H=\epsilon_{0} \rho$, where $\epsilon_{0} \geq 0$ is the proportionality constant $[36,37]$ and $\zeta u_{; i}^{i}=3 \zeta H$. Hence, the effects of both proper pressure and barotropic bulk viscous pressure together can be expressed as

$$
\bar{p}=p-3 \zeta H=\left(\xi-\epsilon_{0}\right) \rho=\epsilon \rho,
$$

where $\epsilon$ is the bulk viscous coefficient. One can infer that such a relation bears similarity to the pressure term with the contribution from perfect fluid $(p=\omega \rho)$, where $\omega$ is the equation of state parameter for perfect fluid. However, a major part of the EoS in $\bar{p}$ of the present model comes from barotropic bulk viscosity. So, in no viscosity condition, the pressure term $\bar{p}$ reduces to the pressure of perfect fluid. Hence, the EMT for viscous fluid is given as

$$
T_{i j}^{\mathrm{vis}}=(\rho+\bar{p}) u_{i} u_{j}-\bar{p} g_{i j}
$$

where $u_{i}$ are the four velocity vectors of the fluid. It may be noted that there are no observational reasons to conclude that pressure is isotropic in DE. However, since the fluids are comoving, one may get this isotropic pressure in DE. Subsequently both the DE fluid and EoS parameter are directiondependent. Hence, the EMT of DE fluid is considered in the form

$$
\begin{aligned}
& T_{i j}^{\mathrm{de}}=\operatorname{diag}\left[\rho^{\mathrm{de}},-p_{x}^{\mathrm{de}},-p_{y}^{\mathrm{de}},-p_{z}^{\mathrm{de}}\right] \\
& =\operatorname{diag}\left[\rho,-\omega_{x}^{\mathrm{de}},-\omega_{y}^{\mathrm{de}},-\omega_{z}^{\mathrm{de}}\right] \rho^{\mathrm{de}} \\
& =\operatorname{diag}\left[1,-\left(\omega^{\mathrm{de}}+\delta\right),-\left(\omega^{\mathrm{de}}+\gamma\right),-\left(\omega^{\mathrm{de}}+\eta\right)\right] \rho^{\mathrm{de}},
\end{aligned}
$$


where $\omega^{\text {de }}$ is the EoS parameter of the DE fluid along the dimensional axes $x, y$, and $z \cdot \rho^{\mathrm{de}}$ is the dark energy density. The deviations of $\omega^{\text {de }}$ from $x$-axis, $y$-axis, and $z$ axis, respectively, denote the skewness parameters $\delta, \gamma$, and $\eta$. In the presence of EMT, Einstein's field equations (2) corresponding to Bianchi type V space-time (1) lead to the following:

$$
\begin{aligned}
& \frac{\ddot{b_{2}}}{b_{2}}+\frac{\ddot{b_{3}}}{b_{3}}+\frac{\dot{b_{2}} \dot{b_{3}}}{b_{2} b_{3}}-\frac{a^{2}}{b_{1}^{2}}=-p+3 \zeta H-\left(\omega^{\mathrm{de}}+\delta\right) \rho^{\mathrm{de}}, \\
& \frac{\ddot{b_{1}}}{b_{1}}+\frac{\ddot{b_{3}}}{b_{3}}+\frac{\dot{b_{1}} \dot{b_{3}}}{b_{1} b_{3}}-\frac{a^{2}}{b_{1}^{2}}=-p+3 \zeta H-\left(\omega^{\mathrm{de}}+\gamma\right) \rho^{\mathrm{de}}, \\
& \frac{\ddot{b_{1}}}{b_{1}}+\frac{\ddot{b_{2}}}{b_{2}}+\frac{\dot{b_{1}} \dot{b_{2}}}{b_{1} b_{2}}-\frac{a^{2}}{b_{1}^{2}}=-p+3 \zeta H-\left(\omega^{\mathrm{de}}+\eta\right) \rho^{\mathrm{de}}, \\
& \frac{\dot{b_{1}} \dot{b_{2}}}{b_{1} b_{2}}+\frac{\dot{b_{2}} \dot{b_{3}}}{b_{2} b_{3}}+\frac{\dot{b_{3}} \dot{b_{1}}}{b_{3} b_{1}}-\frac{3 a^{2}}{b_{1}^{2}}=\rho+\rho^{\mathrm{de}}, \\
& 2 \frac{\dot{b_{1}}}{b_{1}}-\frac{\dot{b_{2}}}{b_{2}}-\frac{\dot{b_{3}}}{b_{3}}=0,
\end{aligned}
$$

where an over dot over the field variable represents the derivatives with respect to the cosmic time $t$. Moreover, the unit of cosmic time is considered as follows: 1 unit of cosmic time $=10$ billion years. The average scale factor $R$ and volumetric scale factor $V$ for the model are, respectively, $R=\left(b_{1} b_{2} b_{3}\right)^{1 / 3}$ and $V=R^{3}=b_{1} b_{2} b_{3}$. The generalized mean Hubble parameter $H$ can be expressed as $H=\dot{R} / R=$ $(1 / 3)\left(H_{x}+H_{y}+H_{z}\right)$, where $H_{x}=\dot{b_{1}} / b_{1}, H_{y}=\dot{b_{2}} / b_{2}$, and $H_{z}=\dot{b}_{3} / b_{3}$ are the directional Hubble parameters in the directions of $x, y$, and $z$, respectively. Now, the field equations (6)-(10) can be framed in Hubble terms as

$$
\begin{aligned}
& \dot{H}_{y}+\dot{H}_{z}+H_{y}^{2}+H_{z}^{2}+H_{y} H_{z}-\frac{a^{2}}{b_{1}^{2}} \\
& =-\bar{p}-\left(\omega^{\mathrm{de}}+\delta\right) \rho^{\mathrm{de}}, \\
& \dot{H}_{x}+\dot{H}_{z}+H_{x}^{2}+H_{z}^{2}+H_{x} H_{z}-\frac{a^{2}}{b_{1}^{2}} \\
& \quad=-\bar{p}-\left(\omega^{\mathrm{de}}+\gamma\right) \rho^{\mathrm{de}}, \\
& \dot{H}_{x}+\dot{H}_{y}+H_{x}^{2}+H_{y}^{2}+H_{x} H_{y}-\frac{a^{2}}{b_{1}^{2}} \\
& \quad=-\bar{p}-\left(\omega^{\mathrm{de}}+\eta\right) \rho^{\mathrm{de}}, \\
& H_{x} H_{y}+H_{y} H_{z}+H_{z} H_{x}-\frac{3 a^{2}}{b_{1}^{2}}=\rho+\rho^{\mathrm{de}}, \\
& 2 H_{x}-H_{y}-H_{z}=0 .
\end{aligned}
$$

The energy conservation equation for viscous fluid, $T_{; j}^{(\mathrm{vis}) i j}=0$, yields

$$
\dot{\rho}+3(\bar{p}+\rho) H=0 .
$$

The energy conservation equation for dark energy fluid, $T_{; j}^{i j(\mathrm{de})}=0$, yields

$$
\begin{aligned}
\dot{\rho}^{\mathrm{de}} & +3 \rho^{\mathrm{de}}\left(\omega^{\mathrm{de}}+1\right) H+\rho^{\mathrm{de}}\left(\delta H_{x}+\gamma H_{y}+\eta H_{z}\right) \\
& =0 .
\end{aligned}
$$

From (16), incorporating the relation between Hubble parameter and average scale factor, we get

$$
\rho=\rho_{0} R^{-3(\epsilon+1)}=\rho_{0}\left(b_{1} b_{2} b_{3}\right)^{-(\epsilon+1)},
$$

where $\rho_{0}$ is the integration constant or rest energy density.

From (14), we have

$$
\rho^{\mathrm{de}}=H_{x} H_{y}+H_{y} H_{z}+H_{z} H_{x}-\frac{3 a^{2}}{b_{1}^{2}}-\rho .
$$

In order to solve (17), we split the conservation equation into two parts: one corresponds to the deviation of equation of the state parameters as $\rho^{\mathrm{de}}\left(\delta H_{x}+\gamma H_{y}+\eta H_{z}\right)=0$ and other is the deviation-free part as $\dot{\rho}^{\mathrm{de}}+3 \rho^{\mathrm{de}}\left(\omega^{\mathrm{de}}+1\right) H=0$ [26]. It can be observed that the behaviour of energy density $\rho^{\mathrm{de}}$ is controlled by the deviation-free part of EoS parameter, whereas anisotropic pressure along different directions can be obtained from second part of the above conservation equation as it corresponds to the conservation of matter field with equal pressure along all directions. Hence, we obtained the dark energy density as

$$
\dot{\rho}^{\mathrm{de}}+3 \rho^{\mathrm{de}}\left(\omega^{\mathrm{de}}+1\right) H=0 \Longrightarrow \rho^{\mathrm{de}}=\rho_{0}^{\mathrm{de}} R^{-3\left(\omega^{\mathrm{de}}+1\right)} .
$$

Now, from (13), incorporating the value of $\eta$, we get

$$
\begin{aligned}
-\omega^{\mathrm{de}} \rho^{\mathrm{de}}= & \left(\frac{H_{y}+H_{z}}{3 H}\right) \frac{\ddot{b_{1}}}{b_{1}}+\left(\frac{H_{x}+H_{z}}{3 H}\right) \frac{\ddot{b_{2}}}{b_{2}} \\
& +\left(\frac{H_{x}+H_{y}}{3 H}\right) \frac{\ddot{b_{3}}}{b_{3}}+\frac{H_{z}}{3 H} \frac{\dot{b_{1}}}{b_{1} b_{2}}+\frac{H_{x}}{3 H} \frac{\dot{b_{2}} \dot{b_{3}}}{b_{2} b_{3}} \\
& +\frac{H_{y}}{3 H} \frac{\dot{b_{1}} \dot{b_{3}}}{b_{1} b_{3}}-\frac{a^{2}}{b_{1}^{2}}+p-3 \zeta H .
\end{aligned}
$$

Again, from (10), with the choice of integrating constant to be unity, we get $b_{1}^{2}=b_{2} b_{3}$. Moreover, for an anisotropic relation, we assume that $b_{2}=b_{3}^{m}$, where $m$ is the average anisotropy parameter [38]. Hence,

$$
b_{1}=b_{3}^{(m+1) / 2} \text {. }
$$

Now, the dark energy density and effective EoS parameter with the function $\Phi(H)=\left(\left(2 \dot{H}+3 H^{2}\right) /(m+1)\right)$ can be reformulated, respectively, as

$$
\begin{aligned}
\rho^{\mathrm{de}} & =\left[2 \frac{\left(m^{2}+4 m+1\right)}{(m+1)^{2}}\right] H^{2}-\frac{3 a^{2}}{b_{1}^{2}}-\rho, \\
\omega^{\mathrm{de}} \rho^{\mathrm{de}} & =-\frac{2}{3}\left(\frac{m^{2}+4 m+1}{m+1}\right) \Phi(H)+\frac{a^{2}}{b_{1}^{2}}-\bar{p} .
\end{aligned}
$$


With the help of (18)-(21), (11)-(13) can be expressed in functional form as

$$
\begin{aligned}
& \gamma=\left(\frac{5+m}{6 \rho^{\mathrm{de}}}\right) \chi(m) F(H), \\
& \eta=-\left(\frac{5 m+1}{6 \rho^{\mathrm{de}}}\right) \chi(m) F(H), \\
& \delta=-\left(\frac{m-1}{3 \rho^{\mathrm{de}}}\right) \chi(m) F(H),
\end{aligned}
$$

where $\chi(m)=(m-1) /(m+1) F(H)=\Phi(H)+3 H^{2} /(m+1)$.

\section{Cosmological Models and Their Behaviours}

From the above formalism, it is quite clear that obtaining an exact solution to the field equations is a cumbersome process. Therefore, without violating any physical meaning of the expression and in order to study the cosmological model in this formalism, we have assumed two scale factors: one leads to power law expansion and the other leads to de Sitter expansion.

3.1. Power Law Expansion Model. Recently, many observational results as well as experiments predict a tensor-toscalar ratio that provides convincing results for standard inflationary scenario even though the value of the ratio contradicts the limits from Planck data. During a power law expansion, the inflationary scenario predicts the generation of gravitational waves. In this model, the scale factor for power law cosmology can be represented as $R=t^{k}$, where $k$ is a positive constant and $k=((m+1) / 2) n$. Also, $m$ and $n$ are positive constants. Now, the volume scale factor $V=t^{3 k}$ and Hubble parameter $H=k / t$. It is obvious that, for $k>1$, the model will be an accelerating one. Now, subsequently, the directional Hubble parameters can be obtained as $H_{x}=((m+$ 1) $/ 2)(n / t), H_{y}=m n / t$, and $H_{z}=n / t$ and consequently the mean deceleration parameter becomes $q=-1+2 / n(m+1)$. The deceleration parameter is a negative constant quantity for $n>2 /(m+1)$, since $m$ and $n$ are positive constants and this is in good agreement with the present observational data that predicts an accelerating universe; therefore, in order to get an accelerating model with this power law scale factor, the exponent $n>1$ if $m<1$; otherwise it has to be decided from $n>2 /(m+1)$.

The universe, in general, is isotropic; but, according to the observational results of CMB temperature anisotropy, a small amount of anisotropy in the universe cannot be ruled out. However, any anisotropy in spatial expansion must be considered as a little perturbation of the isotropic behaviour, which suggests that the exponent $m$ must be close to 1 . In fact, according to the present result from the analysis of anisotropy as predicted from Planck data $[39,40]$ and from our earlier work, $m \approx 1.0001633[21,38]$. The power law model is quite successful in the sense that it neither encounters the horizon problem nor witnesses the flatness problem with $n \geqslant 2 /(m+$
1). The energy density contribution coming from the usual cosmic fluid for the power law model reduces to

$$
\rho=\frac{\rho_{0}}{t^{(3 / 2)(m+1)(\epsilon+1) n}} .
$$

Now, with the help of (26), the dark energy density and dark energy EoS parameter as described in (23) and (24) can be, respectively, reduced to

$$
\begin{aligned}
\rho^{\mathrm{de}} & =\left[\frac{\left(m^{2}+4 m+1\right) n^{2}}{2}\right] \frac{1}{t^{2}}-\frac{3 a^{2}}{t^{n(m+1)}} \\
& -\frac{\rho_{0}}{t^{(3 / 2)(m+1)(\epsilon+1) n}}, \\
\omega^{\mathrm{de}} & =\frac{1}{\rho^{\mathrm{de}}}\left[\left\{\frac{n\left(m^{2}+4 m+1\right)(4-3 n(m+1))}{6(m+1)}\right\} \frac{1}{t^{2}}\right. \\
& \left.+\frac{a^{2}}{t^{n(m+1)}}-\bar{p}\right],
\end{aligned}
$$

where $\bar{p}=\epsilon \rho_{0}^{\mathrm{de}}$. The skewness parameters, $\gamma$, and $\eta$ reduce to

$$
\begin{aligned}
& \gamma=\frac{(5+m)(m-1)}{3(m+1)^{2}}\left(\frac{3 k^{2}-k}{t^{2}}\right) \frac{1}{\rho^{\mathrm{de}}}, \\
& \eta=-\frac{(5 m+1)(m-1)}{3(m+1)^{2}}\left(\frac{3 k^{2}-k}{t^{2}}\right) \frac{1}{\rho^{\mathrm{de}}}, \\
& \delta=-\frac{2(m-1)^{2}}{3(m+1)^{2}}\left(\frac{3 k^{2}-k}{t^{2}}\right) \frac{1}{\rho^{\mathrm{de}}}
\end{aligned}
$$

where $k=n(m+1) / 2$. It is seen that both $\rho$ and $\rho^{\text {de }}$ decrease with the increase in time. The decrease in $\rho^{\text {de }}$ is decided by three different factors, that is, $t^{-2}$ in the first term, $t^{-n(m+1)}$ in the second term, and $t^{3 / 2(1+\epsilon)(m+1) n}$ in the third term. The role of bulk viscous cosmic fluid comes through the third term. One may note that if $\epsilon=-1$, even though the contribution coming from the usual cosmic fluid does not vanish, it does not contribute to the time variation of the dark energy density. For $\epsilon=-1 / 3$, the time variations of second and third terms can be clubbed together. Consequently, for this choice, the skewness parameters become constant quantity and appear to be a simple time-independent deviation from the usual isotropic pressure.

The figures in the manuscript have been drawn for different physical quantities which are expressed in Planckian unit system ( $\left.c=G=k_{B}=h=1\right)$. Also, 1 unit of cosmic time $=10$ billion years. In Figures 1 and 2 , we have observed, respectively, that the matter energy density $\rho$ and the dark energy density $\rho^{\mathrm{de}}$ remain positive during the cosmic evolution for the representative value of the constants ( $m=$ 1.0001633, $n=1.7, a=0.01$, and $\left.\rho_{0}=0.001\right)$. Hence, it indicates that both weak energy condition (WEC) and null energy condition (NEC) are satisfied in the derived model. Further, both $\rho$ and $\rho^{\text {de }}$ decrease with increase in time and slowly reach small positive values in the present epoch. The 


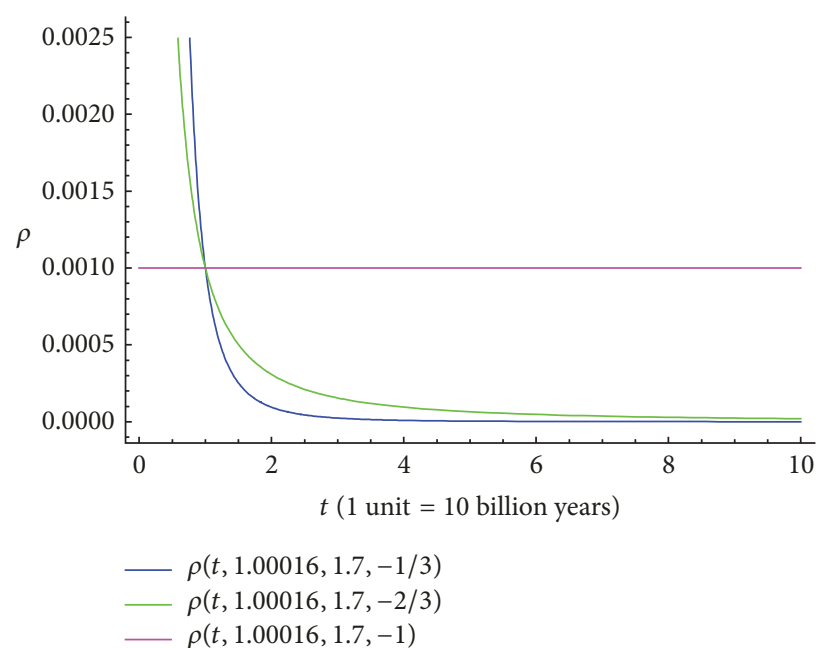

FIgURE 1: Variation of $\rho$ versus $t$ for representative values of the parameters $m=1.0001633, n=1.7$, and $\rho_{0}=0.001$ with different $\epsilon=-1 / 3,-2 / 3,-1$.

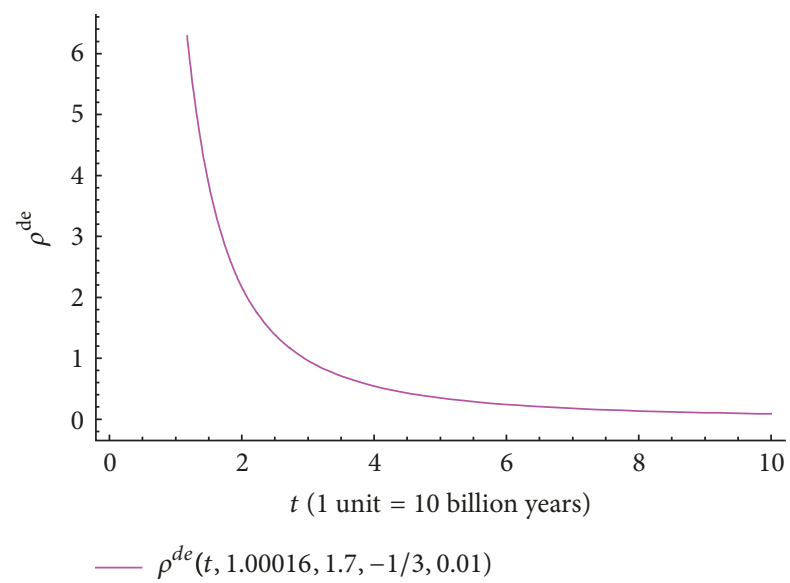

FIGURE 2: Variation of $\rho^{\text {de }}$ versus $t$ for representative values of the parameters $m=1.0001633, n=1.7, a=0.01$, and $\rho_{0}=0.001$ with different $\epsilon=-1 / 3$.

value of dark energy density comes closer to zero and then smoothly approaches small positive value, which indicates that the considered two fluids affect the dark energy density. It is worth noting here that, irrespective of the value of the viscous coefficient $\epsilon$, the behaviour of $\rho^{\mathrm{de}}$ remains alike. So, in Figure 2, we have chosen the value of the viscous coefficient to be $-1 / 3$. However, a small effect of viscous fluid in dark energy density cannot be ruled out. Figure 3 represents the variation of $\omega^{\text {de }}$ with cosmic time for different values of viscous coefficients $\epsilon$. The range value of EoS parameter suggested by combination of SNIa data with CMB anisotropy and galaxy clustering statistics is $[-1.33,-0.79][10]$, whereas the range suggested by recent observations is reduced to more stringent constraints around $-1[34,41,42]$. However, we consider here the earlier data range, since power law behaviour dominates the cosmic dynamics in early phase of cosmic evolution [38]. For $\epsilon=-1 / 3,-2 / 3,-1$, Figure 3

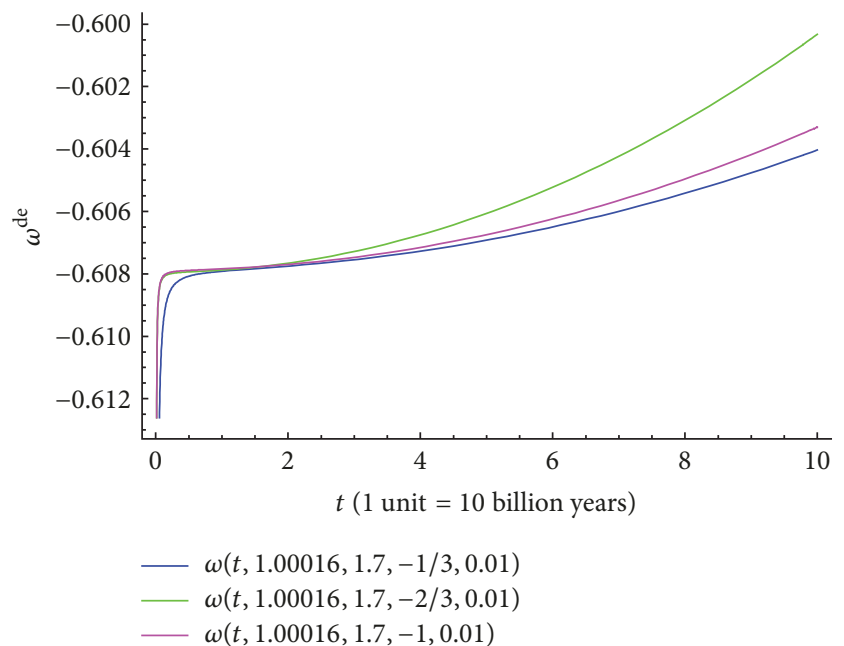

FIGURE 3: Variation of $\omega^{\text {de }}$ versus $t$ for representative values of the parameters $m=1.0001633, n=1.7, a=0.01$, and $\rho_{0}=0.001$ with different $\epsilon=-1 / 3,-2 / 3,-1$.

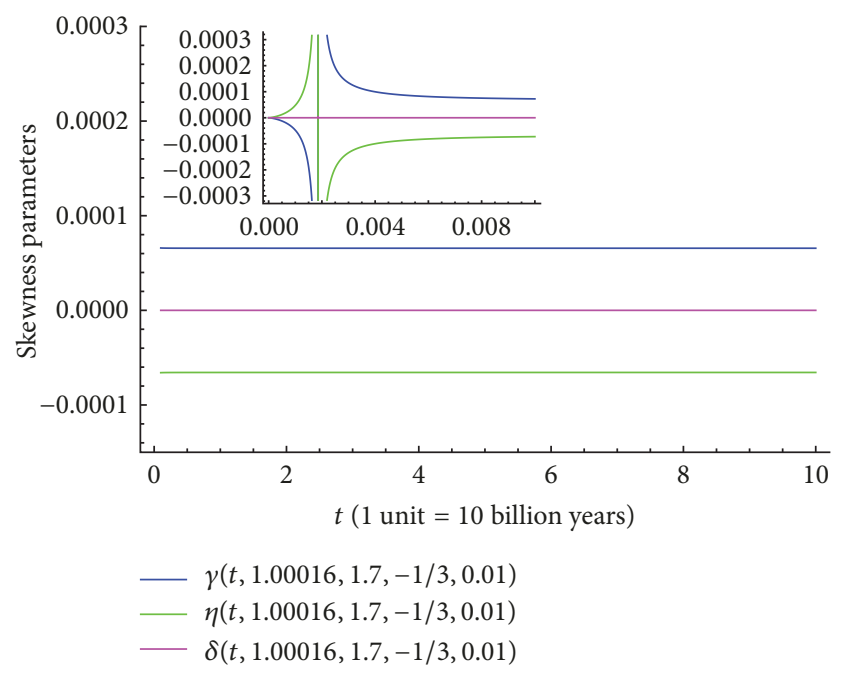

FIgURE 4: Variation of $\delta, \gamma$, and $\eta$ versus $t$ for representative values of the parameters $m=1.0001633, n=1.7, a=0.01$, and $\rho_{0}=0.001$ for $\epsilon=-1 / 3$.

clearly shows that $\omega$ evolves within a range, which is almost aligned with SNIa and CMB observations. Moreover, it is observed that when the bulk viscous coefficient increases, the EoS parameter gradually converges to $\Lambda_{\mathrm{CDM}}$ at late time.

The behaviour of the skewness parameters obtained in (28) has been graphically represented in Figures 4, 5, and 6 , respectively, for $\epsilon=-1 / 3, \epsilon=-2 / 3$, and $\epsilon=-1$. In these figures, it can be noted that the behaviours of skewness parameters are totally controlled by the behaviour of the anisotropic parameter $m[23-25,38]$. For $m=1$, the skewness parameters vanish, giving an indication that the viscous matter affects the skewness parameter. We have observed that, at an early cosmic phase, $\eta$ starts with a negative value far from zero, increases with the cosmic time to become maximum, and then becomes constant with further increase 


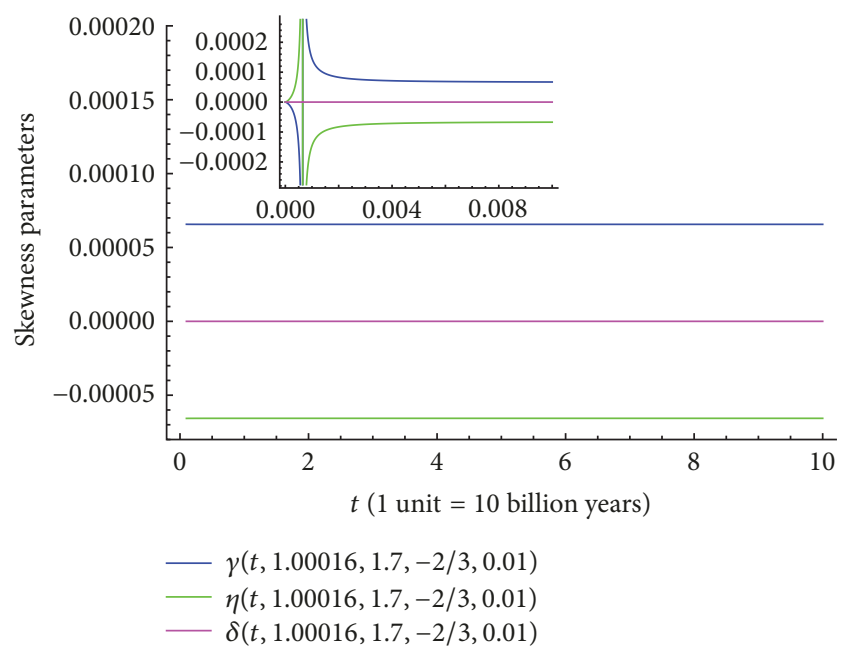

FIGURE 5: Variation of $\delta, \gamma$, and $\eta$ versus $t$ for representative values of the parameters $m=1.0001633, n=1.7, a=0.01$, and $\rho_{0}=0.001$ for $\epsilon=-2 / 3$.

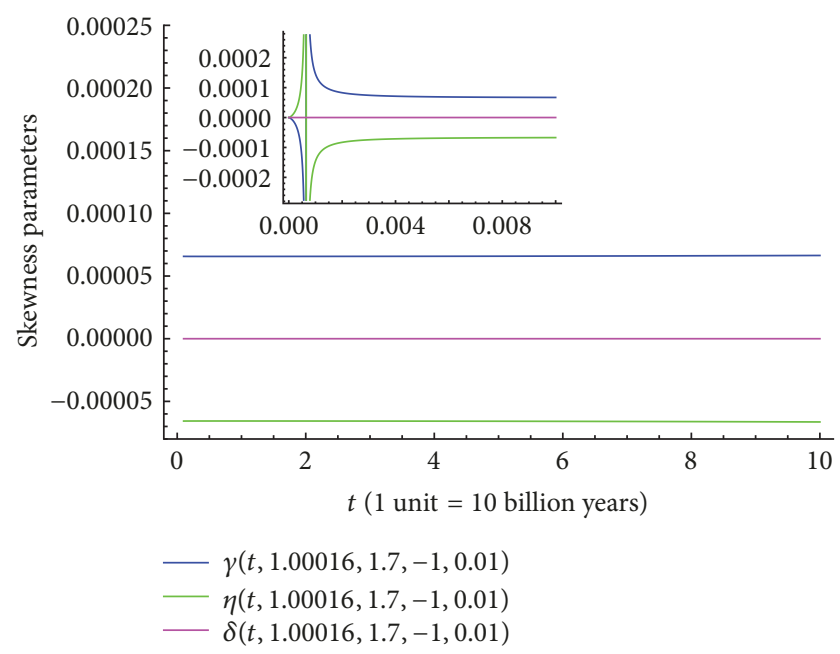

FIGURE 6: Variation of $\delta, \gamma$, and $\eta$ versus $t$ for representative values of the parameters $m=1.0001633, n=1.7, a=0.01$, and $\rho_{0}=0.001$ for $\epsilon=-1$.

in cosmic time. The skewness parameter $\delta$ also starts from a small negative value close to zero and becomes constant with respect to cosmic time. The evolutionary behaviour of $\gamma$ is just the mirror image of $\eta$. It can be noted that the pressure anisotropy factors along $x$-axis, $y$-axis, and $z$-axis $(\eta, \delta$, and $\gamma)$ evolve with different nature, attain their extreme values in a definite range of cosmic time, $0.001<t<0.003$, and remain constant at later time. Therefore, it can be inferred that, in power law cosmology, at an early phase of cosmic evolution, the pressure was assumed to be isotropic; however, in the late phase, pressure anisotropies still remain. It can also be noted that the behaviours of skewness parameters are independent of the choice of the bulk viscous coefficient.

3.2. de Sitter Expansion Model. In de Sitter model, the scale factor is taken as $R=e^{((m+1) / 2) \xi t}$, where $\xi$ is a positive

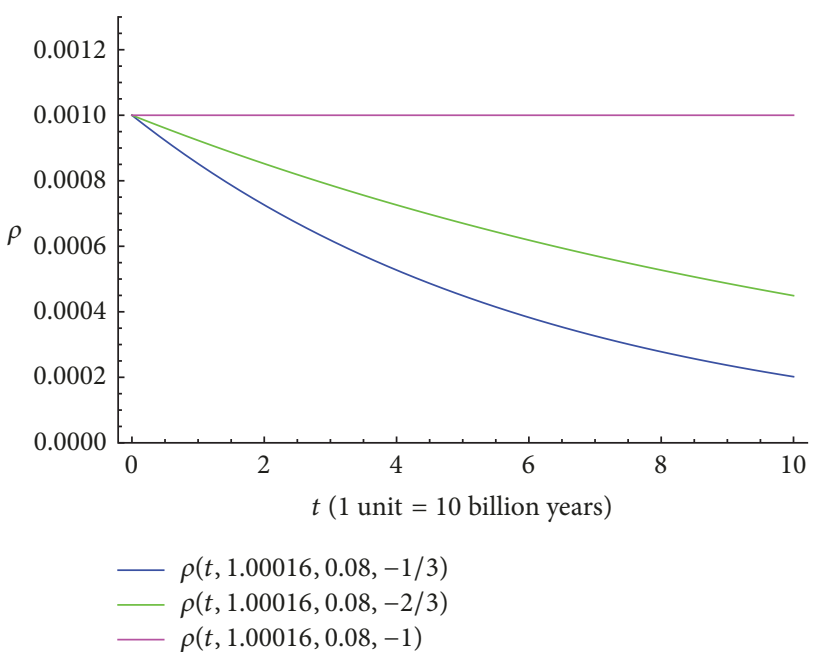

Figure 7: Variation of $\rho$ versus $t$ for representative values of the parameters $m=1.0001633, \rho_{0}=0.001$, and $\xi=0.08$ with different $\epsilon=-1 / 3,-2 / 3,-1$.

constant. In this model, the Hubble parameter is a constant quantity and remains the same throughout the cosmic evolution. The directional Hubble rates along different spatial directions are also constants and can be expressed as $H_{x}=$ $((m+1) / 2) \xi, H_{y}=m \xi$, and $H_{z}=\xi$. So, $\xi$ can be expressed as $\xi=2 H /(m+1)$. With this assumption of the scale factor, the energy density contribution coming from the usual cosmic bulk viscous fluid for the de Sitter model reduces to

$$
\rho=\frac{\rho_{0}}{e^{(3 / 2)(1+\epsilon)(m+1) \xi t}} .
$$

The energy density increases with the decrease in the value of $\epsilon$ and vice versa. For the particular choice $\epsilon=-1, \rho$ becomes independent of time and assumes a constant value $\rho_{0}$ throughout the cosmic evolution (Figure 7).

The rest energy density $\rho^{\text {de }}$ and the dark energy EoS parameter $\omega^{\mathrm{de}}$ for the de Sitter universe can now be obtained as

$$
\begin{aligned}
\rho^{\mathrm{de}}= & \xi^{2}\left(\frac{m^{2}+4 m+1}{2}\right)-\frac{3 a^{2}}{e^{\xi(m+1) t}} \\
& -\frac{\rho_{0}}{e^{(3 / 2)(1+\epsilon)(m+1) \xi t}}, \\
\omega^{\mathrm{de}}= & \frac{1}{\rho^{\mathrm{de}}}\left[-\frac{\left(m^{2}+4 m+1\right) \xi^{2}}{2}+\frac{a^{2}}{e^{\xi(m+1) t}}-\bar{p}\right],
\end{aligned}
$$

where $\bar{p}=\epsilon \rho_{0}^{\mathrm{de}}$. Subsequently, the skewness parameters, $\gamma$, and $\eta$ can be expressed as

$$
\begin{aligned}
& \gamma=\frac{(5+m)(m-1)}{4}\left(\frac{\xi^{2}}{\rho^{\mathrm{de}}}\right), \\
& \eta=-\frac{(5 m+1)(m-1)}{4}\left(\frac{\xi^{2}}{\rho^{\mathrm{de}}}\right), \\
& \delta=-\frac{2(m-1)^{2}}{4}\left(\frac{\xi^{2}}{\rho^{\mathrm{de}}}\right) .
\end{aligned}
$$




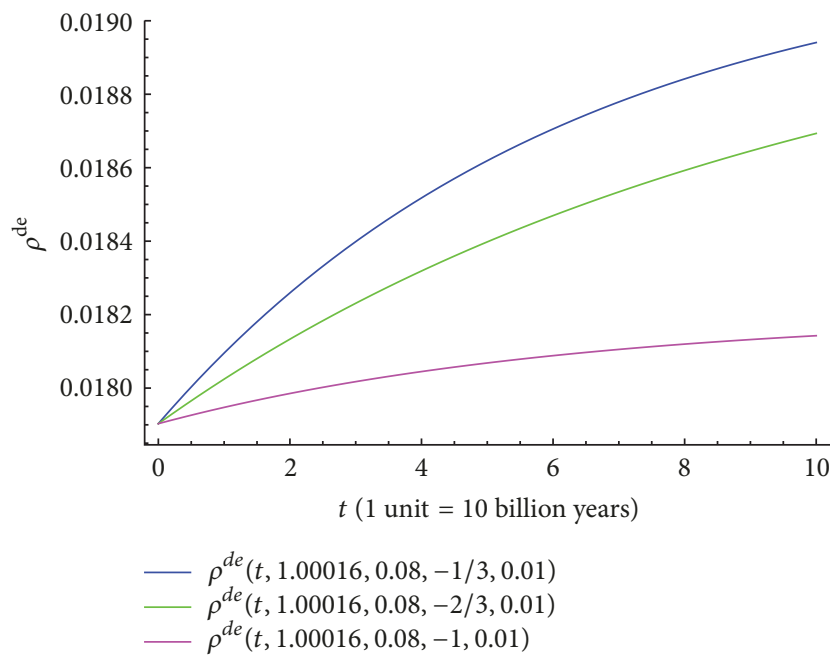

FIGURE 8: Variation of $\rho^{\text {de }}$ versus $t$ for representative values of the parameters $m=1.0001633, \rho_{0}=0.001, a=0.01$, and $\xi=0.08$ with different $\epsilon=-1 / 3,-2 / 3,-1$.

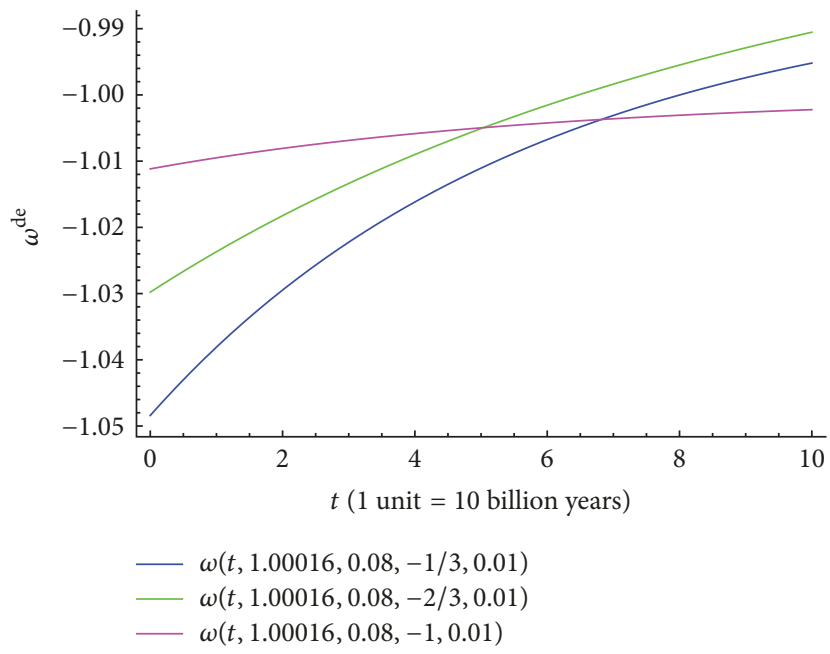

FIGURE 9: Variation of $\omega^{\text {de }}$ versus $t$ for representative values of the parameters $m=1.0001633, \rho_{0}=0.001, a=0.01$, and $\xi=0.08$ with different $\epsilon=-1 / 3,-2 / 3,-1$.

In the de Sitter model, the dark energy density decreases with increase in time and asymptotically reduces to a positive constant. The decrement in $\rho^{\text {de }}$ is decided by four different factors in the second and third terms, namely, $m, \xi, a$, and $\rho_{0}$. The role of bulk viscous cosmic fluid comes through the third term. The contribution from the bulk viscous cosmic fluid becomes time-independent for $\epsilon=-1$. The pressure anisotropies defined earlier in different axis depend on the behaviours of skewness parameters, whereas the dark energy density $\rho^{\text {de }}$ depends on the barotropic equation of state $\epsilon$ (Figure 8).

The EoS parameter in Figure 9 is very much sensitive to the choice of $\epsilon$. For different choices, they start from different values at the early phase and maintain the same evolutionary state at late phase, falling in the observed range as obtained in

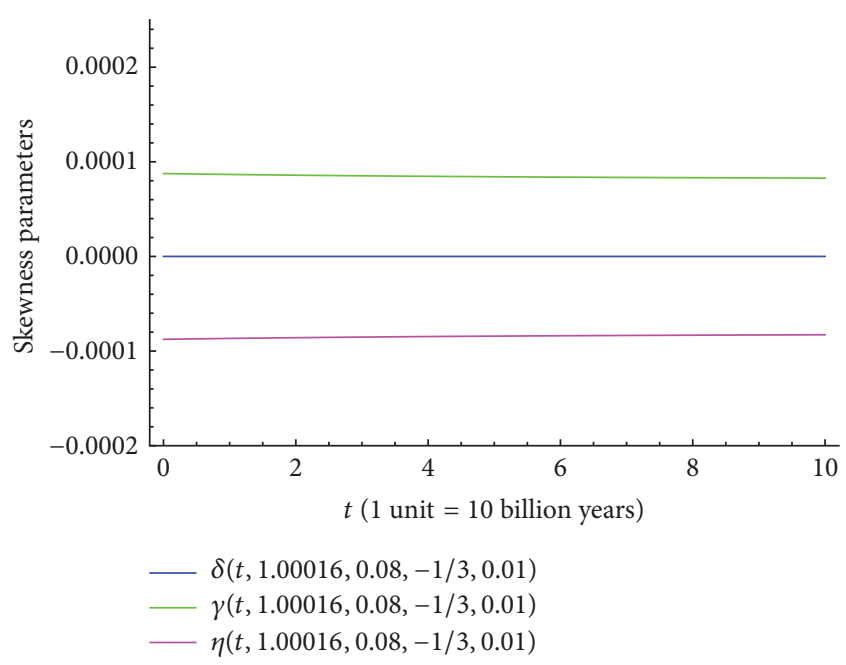

FIGURE 10: Variation of $\delta, \gamma$, and $\eta$ versus $t$ for representative values of the parameters $m=1.0001633, \rho_{0}=0.001, \alpha=0.01$, and $\xi=0.08$ for $\epsilon=-1 / 3$.

2015 Planck data $\omega=-1.019_{-0.080}^{+0.075}$ [34]. The behaviours of EoS parameters are directly proportional to the increasing values of viscous coefficient. At early phase of cosmic evolution, the EoS parameters gathered some amount of energy; however, at late phase, they behave differently. The reason why the dynamics of EoS are greatly affected at early phases is that the bulk viscous fluid has a substantial contribution to the density parameter at that corresponding phase. But, at late phase, the dark energy dominates in spite of the presence of bulk viscous fluid. Hence, cosmic bulk viscous fluid has a very little impact on the dynamics of EoS parameter [37].

The DE skewness parameters are plotted as a function of cosmic time for three representative values of bulk viscous coefficient: $\epsilon=-1,-2 / 3,-1 / 3$. The corresponding skewness parameters are shown for these three viscous coefficients, respectively, in Figures 10, 11, and 12. With increase of viscous coefficient of matter, skewness parameters show nonevolving behaviour in past epoch and rapidly evolve at late phase. The anisotropy in the DE pressure along direction $x$ is almost unaffected by cosmic expansion for all three considered viscous coefficient values. So, the pressure anisotropy vanishes along $x$-axis. $\delta$ 's are less affected by the presence of cosmic fluid compared to $\gamma$ 's and $\eta$ 's. The DE pressures along directions $y$ and $z$ are mostly affected. The reason behind the sensitivity may be due to the consideration of assuming mean Hubble parameter the same as directional Hubble parameter along $x$-axis. At early times, the universe is predicted to have almost isotropic fluid that became anisotropic with the growth of cosmic time. Due to presence of bulk viscous fluid, the anisotropy in DE pressure continues along with the cosmic expansion and decreases slowly at the later period as shown in Figures 10, 11, and 12.

\section{Conclusion}

In the present work, we have investigated the role of anisotropic components on the dynamical aspects of $\mathrm{DE}$ 


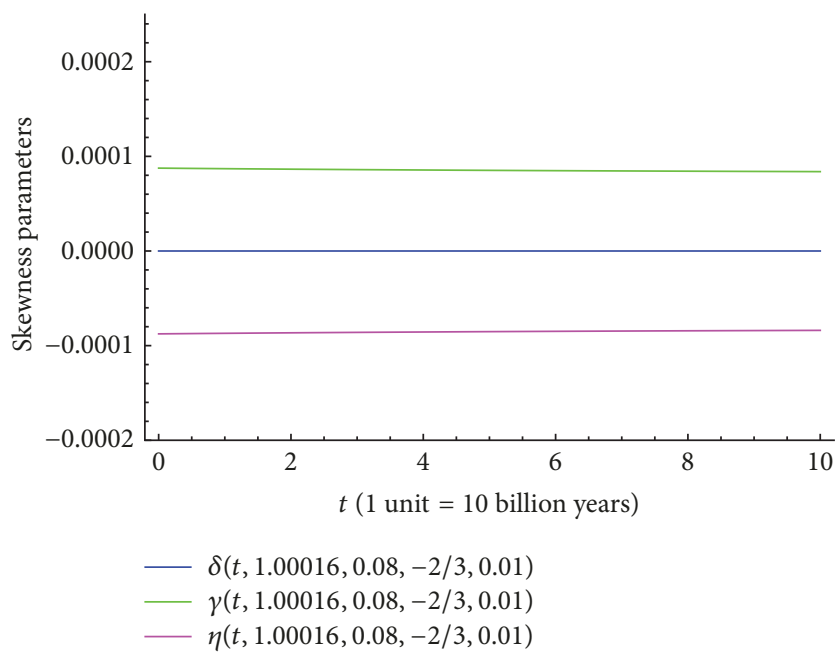

Figure 11: $\delta, \gamma$, and $\eta$ versus $t$ for representative values of the parameters $m=1.0001633, \rho_{0}=0.001, a=0.01$, and $\xi=0.08$ for $\epsilon=-2 / 3$.

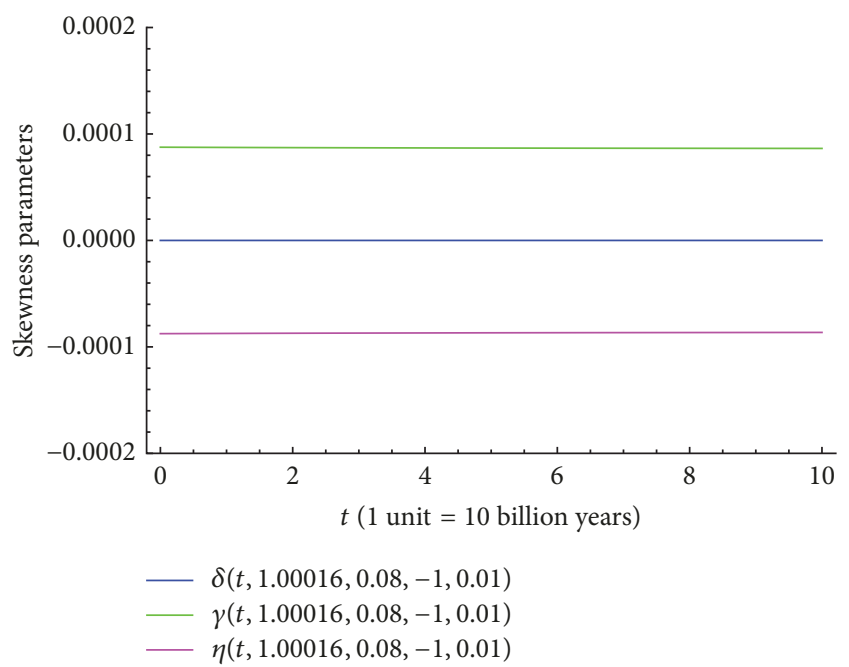

FIGURE 12: $\delta, \gamma$, and $\eta$ versus $t$ for representative values of the parameters $m=1.0001633, \rho_{0}=0.001, a=0.01$, and $\xi=0.08$ for $\epsilon=-1$.

model in Bianchi V space-time in two fluid situations. Two cosmological models have been constructed: one pertaining to power law cosmology and the other pertaining to de Sitter universe. The present model favours a quintessence energy dominated universe in the later universe as $-1<\omega^{\mathrm{de}}<0$; however, in early universe, it favours phantom region. In power law model, EoS parameter lies within the predicted range by observational data. In the de Sitter model, DE dominates at late phase of EoS parameter and bulk viscous fluid plays an important role at early universe. The skewness parameters are dynamically evolving with respect to cosmic expansion. In power law, skewness parameters evolve with different values at early phase, whereas they remain constant at late phase, indicating constant anisotropy rate. However, in de Sitter model, the skewness parameters decrease at the later cosmic period and show a small amount of anisotropy in future cosmic time. The behaviours of skewness parameters are independent of the choice of the bulk viscous coefficient. There are a lot of observational evidences to support $\Lambda_{\mathrm{CDM}}$, such as $\mathrm{CMB}$ and redshift-distance relation. However, our considered model is a generalization of FRW model or $\Lambda_{\mathrm{CDM}}$ model as discussed in Section 2. Also, our model is scalefactor-dependent and may change its behaviour in different scale factors; however, the formalism developed here clearly indicates the accelerating behaviour of the expanding universe. Moreover, there is resemblance of data of considered model with standard $\Lambda_{\mathrm{CDM}}$ model; our model found here also aligned with the present day observational outcomes.

There are ways to know whether the model approaching to $\Lambda_{\mathrm{CDM}}$ model is accurate or not. One way is that $\Lambda_{\mathrm{CDM}}$ universes may converge to de Sitter universe under special conditions as de Sitter model has the exponential growth. In our investigation also, the EoS parameter in de Sitter universe is similar to EoS parameter of $\Lambda_{\mathrm{CDM}}$ model at late cosmic time of evolution. Another way is the state finder diagnostics, which checks the validity of the model. $\Lambda_{\mathrm{CDM}}$ model has state finder pair $(r, s)=(1,0)$. The model discussed here also confirms the acceptability by state finder analysis with the pair $(1,0)$ both for the de Sitter universe and for power law for large value of the exponent $m$.

\section{Conflicts of Interest}

The authors declare that they have no conflicts of interest.

\section{Acknowledgments}

B. Mishra and Pratik P. Ray acknowledge DST, New Delhi, India, for providing facilities through DST-FIST Lab, Department of Mathematics, where a part of this work was done. S. K. J. Pacif acknowledges NBHM, Department of Atomic Energy (DAE), Government of India, for the postdoctoral fellowship.

\section{References}

[1] A. G. Riess, A. V. Filippenko, P. Challis et al., "Observational evidence from supernovae for an accelerating universe and a cosmological constant," The Astronomical Journal, vol. 116, no. 3, pp. 1009-1038, 1998.

[2] S. Perlmutter, G. Aldering, and G. Goldhaber, "Measurements of $\Omega$ and $\Lambda$ from 42 high-redshift supernovae," The Astrophysical Journal, vol. 517, no. 2, pp. 565-586, 1999.

[3] P. A. R. Ade et al., "Planck collaboration XV, planck 2013 results. XV. CMB power spectra and likelihood ," Astronomy Astrophys, vol. 571, p. A15, 2014.

[4] P. A. R. Ade et al., "Results. XI. CMB power spectra, likelihoods and robustness of parameters," Astronomy Astrophys, vol. 594, A11, 2015.

[5] L. Pogosian and T. Vachaspati, "Cosmic microwave background anisotropy from wiggly strings," Physical Review D: Particles, Fields, Gravitation and Cosmology, vol. 60, no. 8, 1999.

[6] P. A. R. Ade et al., "Planck Collaboration XVII, Planck 2013 results. XVII. Gravitational lensing by large-scale structure," Astronomy Astrophys, vol. 571, p. A17, 2013. 
[7] P. A. R. Ade et al., "Planck 2015 cosmological results Planck Collaboration XV, Planck 2015 results. XV. Gravitational lensing," Astronomy Astrophys, vol. 594, p. A15, 2016.

[8] E. J. Copeland, M. Sami, and S. Tsujikawa, "Dynamics of dark energy," International Journal of Modern Physics D, vol. 15, no. 11, pp. 1753-1935, 2006.

[9] M. Li, X. D. Li, S. Wang, and Y. Wang, "Dark energy," Communications in Theoretical Physics, vol. 56, no. 3, p. 525, 2011.

[10] S. Kumar and A. K. Yadav, "Some bianchi type-v models of accelerating universe with dark energy," Modern Physics Letters A, vol. 26, no. 9, pp. 647-659, 2011.

[11] J. D. Barrow, "Cosmological limits on slightly skew stresses," Physical Review D: Particles, Fields, Gravitation and Cosmology, vol. 55, no. 12, pp. 7451-7460, 1997.

[12] J. D. Barrow and R. Maartens, "Anisotropic stresses in inhomogeneous universes," Physical Review D: Particles, Fields, Gravitation and Cosmology, vol. 59, no. 4, 043502, 7 pages, 1999.

[13] T. Abbott et al., "The Dark Energy Survey: more than dark energy - an overview," Monthly Notices of the Royal Astronomical Society, vol. 460, no. 2, pp. 1270-1299, 2016.

[14] P. A. R. Ade, V. Aghanim, M. Arnaud et al., "Planck Collaboration XIV, Planck 2015 results. XIV. Dark energy and modified gravity," Astronomy Astrophys, vol. 594, p. A14, 2016.

[15] M. Cruz, L. Cayón, E. Martínez-González, P. Vielva, and J. Jin, "The non-gaussian cold spot in the 3 year Wilkinson microwave anisotropy probe data," The Astrophysical Journal, vol. 655, no. 1 I, pp. 11-20, 2007.

[16] J. Hoftuft, H. K. Eriksen, A. J. Banday, K. M. Górski, F. K. Hansen, and P. B. Lilje, "Increasing evidence for hemispherical power asymmetry in the five-year WMAP data," The Astrophysical Journal, vol. 699, no. 2, pp. 985-989, 2009.

[17] C. L. Bennett et al., "Seven-year wilkinson microwave anisotropy probe (wmap) observations: are there cosmic microwave background anomalies?" The Astrophysical Journal Supplement Series, vol. 192, no. 17, 2011.

[18] D. N. Spergel, R. Bean, and O. Doré, “Three-year wilkinson microwave anisotropy probe (wmap) observations: implications for cosmology," The Astrophysical Journal Supplement Series, vol. 170, no. 2, article 377, 2007.

[19] E. Komatsu et al., "Seven-year wilkinson microwave anisotropy probe (wmap) observations: cosmological interpretation," The Astrophysical Journal Supplement Series, vol. 192, no. 18, 2011.

[20] L. Campanelli, P. Cea, G. L. Fogli, and A. Marrone, "Testing the isotropy of the Universe with type Ia supernovae," Physical Review D: Particles, Fields, Gravitation and Cosmology, vol. 83, no. 10, Article ID 103503, 2011.

[21] B. Mishra, P. K. Sahoo, and S. K. Tripathy, "Pressure anisotropy and dark energy models in scale invariant theory of gravitation," Astrophysics and Space Science, vol. 356, no. 1, pp. 163-171, 2015.

[22] B. Mishra, P. P. Ray, and S. K. Pacif, "Dark energy cosmological models with general forms of scale factor," The European Physical Journal Plus, vol. 132, no. 10, 2017.

[23] S. K. Tripathy, B. Mishra, and P. K. Sahoo, "Two fluid anisotropic dark energy models in a scale invariant theory," The European Physical Journal Plus, vol. 132, no. 9, 2017.

[24] B. Mishra, P. K. Sahoo, and P. P. Ray, "Accelerating dark energy cosmological model in two fluids with hybrid scale factor," International Journal of Geometric Methods in Modern Physics, vol. 14, no. 9, 1750124, 15 pages, 2017.

[25] B. Mishra, S. K. Tripathy, and P. R. Pratik, "Anisotropy in dark Energy," physics. General Physics, 2017.
[26] O. Akarsu and C. B. Kilinc, "LRS Bianchi type I models with anisotropic dark energy and constant deceleration parameter," General Relativity and Gravitation, vol. 42, no. 1, pp. 119-140, 2010.

[27] O. Akarsu and C. B. Kilinc, "Bianchi type III models with anisotropic dark energy," General Relativity and Gravitation, vol. 42, no. 4, pp. 763-775, 2010.

[28] A. K. Yadav, F. Rahaman, and S. Ray, "Dark energy models with variable equation of state parameter," International Journal of Theoretical Physics, vol. 50, no. 3, pp. 871-881, 2011.

[29] H. Amirhashchi, "Phantom instability of viscous dark energy in anisotropic space-time," Astrophysics and Space Science, vol. 345, no. 2, pp. 439-447, 2013.

[30] A. Pradhan, H. Amirhashchi, and B. Saha, "An interacting and non-interacting two-fluid scenario for dark energy in FRW universe with constant deceleration parameter," Astrophysics and Space Science, vol. 333, no. 1, pp. 343-350, 2011.

[31] A. Sheykhi and M. R. Setare, "Thermodynamical description of the interacting new agegraphic dark energy," Modern Physics Letters A, vol. 26, no. 25, pp. 1897-1907, 2011.

[32] R. M. Wald, General Relativity, University of Chicago Press, Chicago, IL, USA, 1984.

[33] T. R. Jaffe, S. Hervik, A. J. Banday, and K. M. Gorski, "On the viability of bianchi type $\mathrm{VII}_{H}$ models with dark energy," The Astrophysical Journal, vol. 644, no. 2, pp. 701-708, 2006.

[34] P. A. R. Ade, N. Aghanim, M. Arnaud et al., "Planck 2015 results. XIII. Cosmological Parameters," Astronomy Astrophysics, vol. 594, p. A13, 2016.

[35] A. Pontzen and A. Challinor, "Bianchi model CMB polarization and its implications for CMB anomalies," Monthly Notices of the Royal Astronomical Society, vol. 380, no. 4, pp. 1387-1398, 2007.

[36] S. K. Tripathy, D. Behera, and T. R. Routray, "Anisotropic universe with cosmic strings and bulk viscosity," Astrophysics and Space Science, vol. 325, no. 1, pp. 93-97, 2009.

[37] I. Brevik, "Viscosity-induced crossing of the phantom barrier," Entropy, vol. 17, no. 9, pp. 6318-6328, 2015.

[38] B. Mishra and S. K. Tripathy, "Anisotropic dark energy model with a hybrid scale factor," Modern Physics Letters A, vol. 30, no. 36, Article ID 1550175, 2015.

[39] P. A. Ade, R. N. Aghanim, C. Armitage-Caplan, M. Arnaud, M. Ashdown, and F. Atrio-Barandela, "Planck collaboration xxiii, planck 2013 results. xxiii, isotropy and statistics of the cmb," Astronomy Astrophysics, vol. 571, p. A23, 2014.

[40] P. A. R. Ade, N. Aghanim, Y. Akrami et al., "Planck collaboration xvi, planck 2015 results. xvi, isotropy and statistics of the cmb," Astronomy Astrophysics, vol. 594, p. A16, 2016.

[41] P. A. R. Ade, N. Aghanim, and C. Armitage-Caplan, "Planck 2013 results. XVI. Cosmological parameters," Astronomy Astrophysics, vol. 571, A16, 2014.

[42] M. Betoule et al., "Improved cosmological constraints from a joint analysis of the SDSS-II and SNLS supernova samples," Astronomy Astrophysics, vol. 568, no. A22, 2014. 

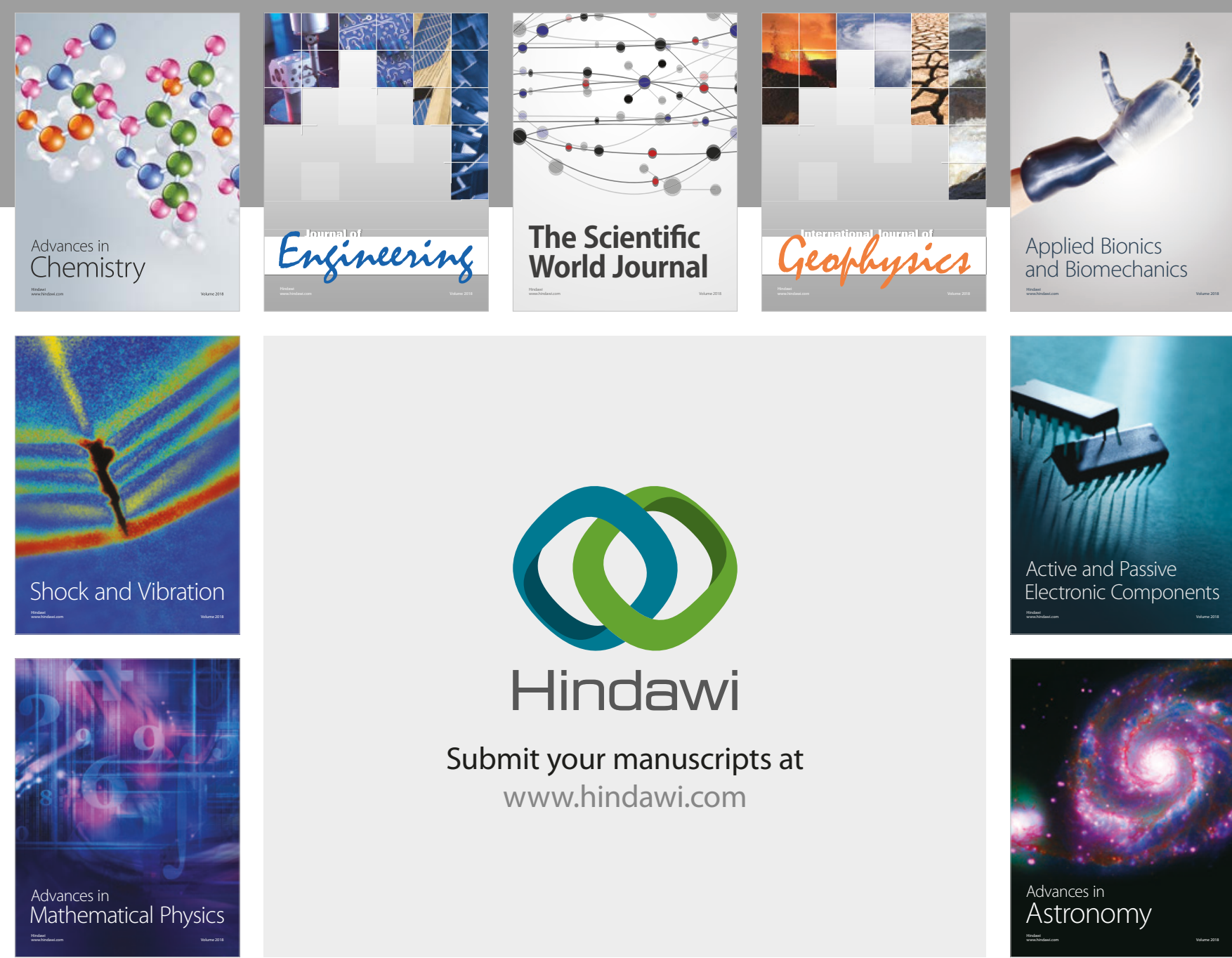

Submit your manuscripts at

www.hindawi.com

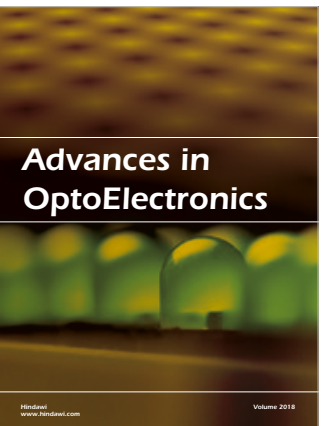

\section{Rotcting Machinery}
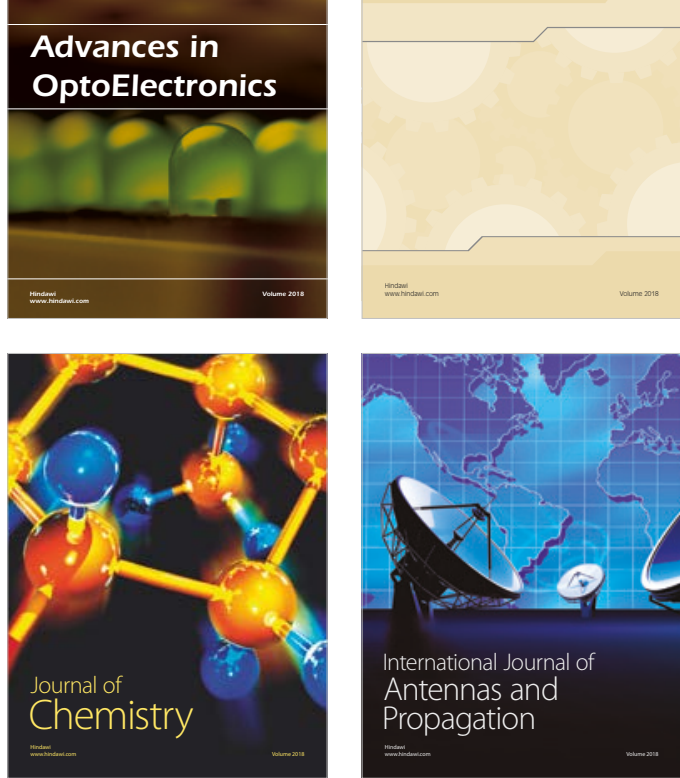

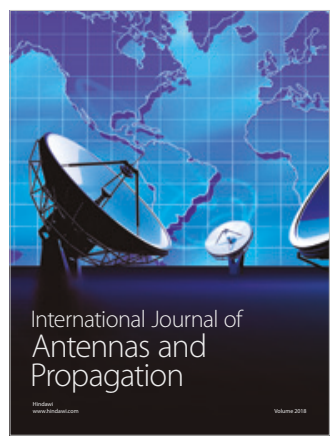

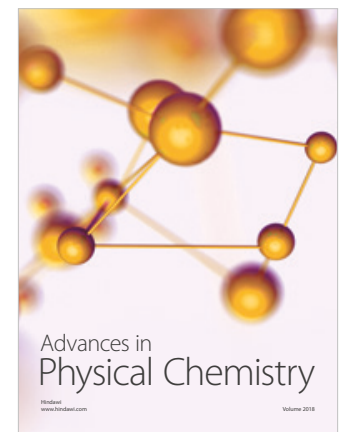

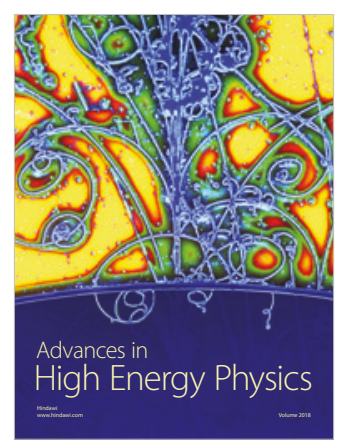

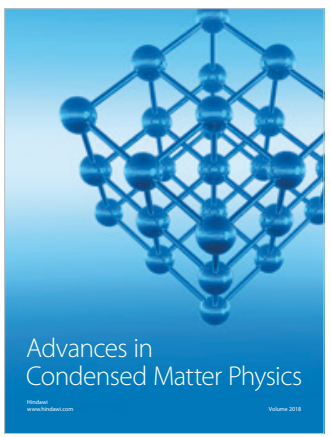

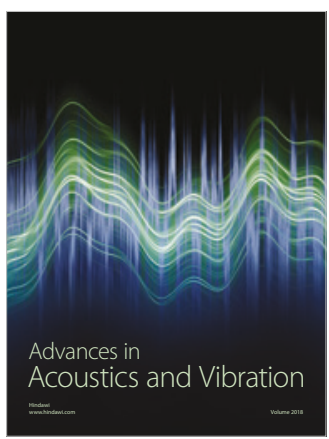

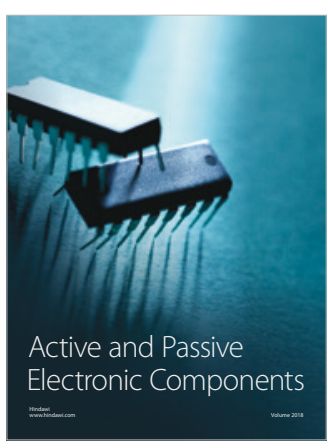
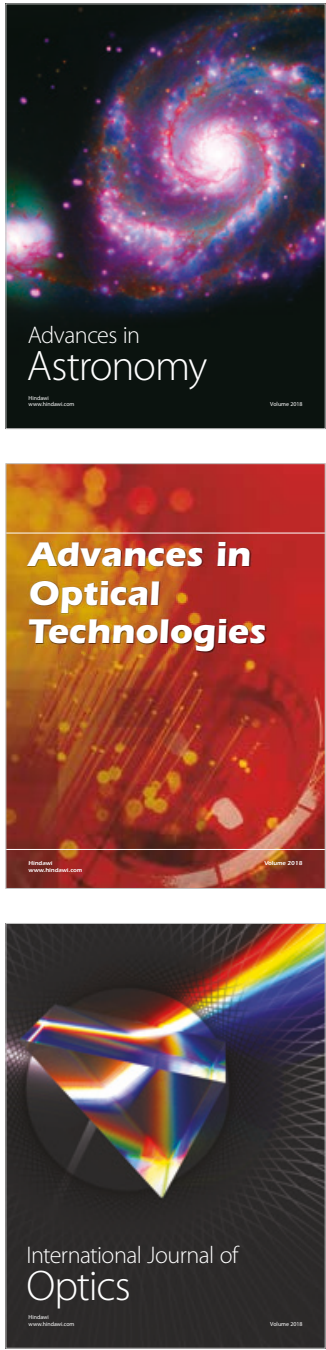